\title{
ON A QUESTION OF MILNOR CONCERNING SINGULARITIES OF MAPS
}

\author{
ELMER G. REES \\ Department of Mathematics and Statistics, James Clerk Maxwell Building, \\ King's Buildings, Edinburgh EH9 3JZ, UK
}

(Received 20 April 1998)

\begin{abstract}
Restrictions are given on the dimensions $m$ and $k$ for which there is a map $f: \mathbb{R}^{m} \rightarrow \mathbb{R}^{k}$ whose Jacobian has rank $k$ in a neighbourhood of a singular point if $f$ is either quadratic or even. The restrictions are shown to be best possible in the quadratic case.
\end{abstract}

Keywords: singularities; real polynomials; Milnor's hypothesis; non-singular real matrices

AMS 1991 Mathematics subject classification: Primary 32S55; $15 \mathrm{~A} 03$

\section{Preamble}

Chapter 11 of [8] discusses the class of polynomial maps $f: \mathbb{R}^{m} \rightarrow \mathbb{R}^{k}$ that satisfy what we will call Milnor's hypothesis.

Milnor's hypothesis. The derivative $\mathrm{d} f_{x}: \mathbb{R}^{m} \rightarrow \mathbb{R}^{k}$ of $f$ at $x$ has rank $k$ for every $x$ in some punctured neighbourhood of $0 \in \mathbb{R}^{m}$, but $\mathrm{d} f_{0}$ has rank less than $k$.

Theorem 11.1 of [8] shows that functions that satisfy the hypothesis have several of the nice geometric properties enjoyed by complex analytic maps $\mathbb{C}^{m} \rightarrow \mathbb{C}$ having an isolated critical point at 0 . However, Milnor also pointed out that it is not easy to find other maps that satisfy the hypothesis, although he gave some examples of such quadratic functions constructed using the quaternions and Cayley numbers. Since then, a number of authors have found interesting examples when $k=2$ (see, for example, $[7,9,13]$ ).

The object of this note is to give restrictions on the possible values of $m$ and $k$ for which maps satisfying Milnor's hypothesis can occur, at least for the case where the map $f$ is given by quadratic polynomials or by an even function. Milnor conjectured that there are no 'non-trivial' examples for $m<2(k-1)$. A more precise definition of 'non-trivial' is given in [7]. Under certain conditions, Theorems 1.1 and 1.2 show that there are no examples at all in a rather wider range of dimensions, but we give examples to show that some of the results here are best possible. The method is to reduce the problem to one closely related to the vector fields on spheres problem solved by Adams in [1]. Indeed, 
results similar to Theorems 1.1 and 1.2 can be deduced from Adams' main theorem, the precise relationship is discussed in [11] and [12].

Let $\rho(m)$ denote the Radon-Hurwitz number (see $[1,4,10]$ ), which can be defined as

(i) $\rho(m n)=\rho(m)$ if $n$ is odd;

(ii) $\rho(m)=m$ if $m=1,2,4,8$; and

(iii) $\rho(16 m)=\rho(m)+8$ for $m \geqslant 1$.

Theorem 1.1. If $f: \mathbb{R}^{m} \rightarrow \mathbb{R}^{k}$ is an even function that satisfies Milnor's hypothesis, then $k \leqslant \rho(m)$.

In the special case $k=2$, this implies that $m$ must be even and, more generally, that $m$ must be divisible by a certain power of 2 that increases with $k$.

In general, the result of Theorem 1.1 cannot be improved; for example, a quadratic map $\mathbb{R}^{16} \rightarrow \mathbb{R}^{9}$ is described in [8], it satisfies the hypothesis and $\rho(16)=9$. Maps with similar properties in other dimensions are constructed below using formulae based on Clifford algebras. It is natural to start by considering maps of low degree. Theorem 1.2 gives the best possible dimensions for the existence of quadratic maps satisfying Milnor's hypothesis. In some cases (if $m=2^{s} n$ with $n$ odd and $s \equiv 2,3 \bmod 4$ ), Theorem 1.2 gives more severe restrictions on the dimension than does Theorem 1.1.

Theorem 1.2. If $f: \mathbb{R}^{m} \rightarrow \mathbb{R}^{k}$ is a quadratic function that satisfies Milnor's hypothesis, then $k \leqslant \rho(m / 2)+1$. (If $m$ is odd, then $\rho(m / 2)=0$.)

Proof of Theorem 1.1. Choose a small sphere $S_{\epsilon}$ centred at $0 \in \mathbb{R}^{m}$, such that $\mathrm{d} f_{x}$ has rank $k$ for each $x \in S_{\epsilon}$. The map $x \rightarrow \mathrm{d} f_{x}$ defines

$$
S_{\epsilon} \rightarrow \operatorname{Lin}\left(\mathbb{R}^{m}, \mathbb{R}^{k}\right) \text {. }
$$

Since $f$ is an even function, one has that $\mathrm{d} f_{-x}=-\mathrm{d} f_{x}$. Now consider the map

$$
e: S_{\epsilon} \times \mathbb{R}^{m} \rightarrow S_{\epsilon} \times \mathbb{R}^{k}
$$

defined by evaluation, i.e. $e(x, y)=\left(x, \mathrm{~d} f_{x}(y)\right)$.

Let $\tau_{1}$ be the involution defined on $S_{\epsilon} \times \mathbb{R}^{m}$ by $\tau_{1}(x, y)=(-x,-y)$ and $\tau_{2}$ be the involution defined on $S_{\epsilon} \times \mathbb{R}^{k}$ by $\tau_{2}(x, y)=(-x, y)$. Then $e$ is equivariant with respect to the involutions $\tau_{1}, \tau_{2}$, and so, by its linearity properties, it defines a map of vector bundles

$$
S_{\epsilon} \times_{\tau_{1}} \mathbb{R}^{m} \rightarrow S_{\epsilon} \times_{\tau_{2}} \mathbb{R}^{k} \cong\left(S_{\epsilon} / \pm 1\right) \times \mathbb{R}^{k} .
$$

By the hypothesis this map is onto each fibre. Hence, by the splitting of epimorphisms of vector bundles, the bundle $m \lambda$ over $\mathbb{R}^{m-1}$ contains a trivial $k$-dimensional bundle. Theorem 1.1 now follows from a refinement of Adams's solution [1] to the vector fields on spheres problem, due to several authors (for example, Theorem 2.4 of [14] or Theorem 1.10 of [3]). Yoshida's proof is as follows. 
If $m$ is even, the result follows by noting that the Stiefel Whitney class $w_{m-1}(m \lambda)$ is non-zero. If $m$ is odd, we already have that $m \lambda$ is isomorphic to $\eta \oplus \epsilon$, where $\epsilon$ denotes trivial line bundle and $\eta$ has $k-1$ sections. Corollary 1.11 of [5] shows that $\eta$ is isomorphic to the tangent bundle of $\mathbb{R}^{m-1}$ and so, by the main result of $[\mathbf{1}], \eta$ has at most $\rho(m)-1$ sections, as required.

Proof of Theorem 1.2. First we show that the linear part of such a quadratic map must be zero. The following result is partly due to Santiago Lopez de Medrano who proved it in the case $m=k$.

Proposition 1.3. If $f: \mathbb{R}^{m} \rightarrow \mathbb{R}^{k}$ is a function that satisfies Milnor's hypothesis and each of its components has the form

$$
\frac{1}{2} x^{\mathrm{T}} A x+a^{\mathrm{T}} x,
$$

where $A$ is a symmetric $m \times m$ real matrix and $a \in \mathbb{R}^{m}$, then each a vanishes, i.e. $f$ is purely quadratic.

Proof. Milnor's hypothesis is that the derivative $\mathrm{d} f_{x}$ has rank $k$ for all small, non-zero vectors $x$ but has smaller rank for $x=0$. Clearly,

$$
\mathrm{d} f_{x}=\left(x^{\mathrm{T}} A_{1}+a_{1}^{\mathrm{T}}, x^{\mathrm{T}} A_{2}+a_{2}^{\mathrm{T}}, \ldots, x^{\mathrm{T}} A_{k}+a_{k}^{\mathrm{T}}\right) .
$$

Since the rank is less than $k$ at $x=0$, the vectors $a_{1}, a_{2}, \ldots, a_{k}$ are linearly dependent, and so we can assume, by using a change of basis in $\mathbb{R}^{k}$, that $a_{1}=0$. We can also assume that $a_{2} \neq 0$, since, if all the $a_{r}=0$, the result would be true. It is also true that $A_{1}$ is non-singular, since, if not, there would be arbitrarily small non-zero vectors $x$ in the kernel of $A_{1}$ and then, for these $x, \mathrm{~d} f_{x}$ has rank $<k$.

So, we can assume that $a_{1}=0, a_{2} \neq 0$ and $A_{1}$ is non-singular. For large values of $\lambda$, the matrix $A_{2}-\lambda A_{1}$ is non-singular, so there is a vector $x_{\lambda}$ such that

$$
\left(A_{2}-\lambda A_{1}\right) x_{\lambda}=-a_{2} .
$$

As $\lambda \rightarrow \infty, x_{\lambda} \rightarrow 0$ and the rank of $\mathrm{d} f_{x_{\lambda}}$ is less than $k$ for each $\lambda$; which contradicts Milnor's hypothesis.

To continue with the proof, note that the derivative of $f$ gives a map

$$
\mathrm{d} f: \mathbb{R}^{m} \rightarrow \operatorname{Lin}\left(\mathbb{R}^{m}, \mathbb{R}^{k}\right) .
$$

If $f(x)=\frac{1}{2}\left(x^{\mathrm{T}} A_{1} x, x^{\mathrm{T}} A_{2} x, \ldots, x^{\mathrm{T}} A_{k} x\right)$, where the $A_{r}$ are symmetric $m \times m$ real matrices, then $\mathrm{d} f_{x}$ is given by the matrix $\left[x^{\mathrm{T}} A_{1}, x^{\mathrm{T}} A_{2}, \ldots, x^{\mathrm{T}} A_{k}\right]$. If this is non-singular for every choice of non-zero $x$, then the non-zero elements of the linear subspace (of the space of real symmetric $m \times m$ matrices) whose basis is $\left\{A_{1}, A_{2}, \ldots, A_{k}\right\}$ are all non-singular. Hence, by the main result of [2], one has that $k-1 \leqslant \rho(m / 2)$. 
Theorem 1.2 is best possible in the sense that for every $m, k$ such that $k=\rho(m / 2)+1$, there is a quadratic map $f: \mathbb{R}^{m} \rightarrow \mathbb{R}^{k}$ that satisfies Milnor's hypothesis. The required maps can be described by a number of constructions (see, for example, $[2,6]$ ). Following the ideas of [6], consider (with $m=2 r$ ) a set $\left\{\gamma_{1}=I, \gamma_{2}, \ldots, \gamma_{\ell}\right\}$ of $r \times r$ real orthogonal transformations that satisfies the following identities:

$$
\gamma_{i}^{2}=-I \text { for } 2 \leqslant i \leqslant \ell \text { and } \gamma_{i} \gamma_{j}+\gamma_{j} \gamma_{i}=0 \text { for } 2 \leqslant i<j
$$

Using these relations, one can see that such a set is a basis for a space of $r \times r$ real matrices in which every non-zero element has maximal rank, and, hence, $\ell \leqslant \rho(r)$. Such matrices generate a Clifford algebra with negative definite quadratic form. Lam's explicit construction of a suitable set of matrices $\gamma_{i}$ with $\ell=\rho(r)$ is described with the present notation in [12].

Now, following [2], let $C_{0}$ be the $2 r \times 2 r$ matrix

$$
\left[\begin{array}{cc}
I & 0 \\
0 & -I
\end{array}\right]
$$

and, for $i \geqslant 1$, let $C_{i}$ be the $2 r \times 2 r$ (symmetric) matrix

$$
\left[\begin{array}{cc}
0 & \gamma_{i} \\
\gamma_{i}^{\mathrm{T}} & 0
\end{array}\right]
$$

The required map $f: \mathbb{R}^{2 r} \times \mathbb{R}^{2 r} \rightarrow \mathbb{R}^{\ell+1}$ is given by

$$
f(x, y)=\left(x^{\mathrm{T}} C_{0} y, x^{\mathrm{T}} C_{1} y, x^{\mathrm{T}} C_{2} y, \ldots, x^{\mathrm{T}} C_{\ell} y\right) .
$$

Acknowledgements. This work was started during a visit to the Instituto de Matematicas, UNAM, Cuernavaca, Mexico. I am very grateful to Dr José A. Seade for organizing the visit, to the Instituto for support, and to the Royal Society for a travel grant. I am particularly grateful to him for bringing Milnor's question to my attention.

I also thank Dr Santiago Lopez de Medrano for his interest in the problem and in particular for pointing out the Proposition.

Finally, Professor Donald M. Davis was kind enough to point out that he had proved the result needed to complete the proof of Theorem 1.1 and provided me with the reference [14].

\section{References}

1. J. F. AdaMs, Vector fields on spheres, Ann. Math. 75 (1962), 603-632.

2. J. F. ADAMS, P. LAX AND R. PHILliPS, On matrices whose real linear combinations are non-singular, Proc. Am. Math. Soc. 16 (1965), 318-322.

3. D. M. DavIS, Desuspensions of stunted projective spaces, Pacific J. Math. 113 (1984), 35-49.

4. A. HuRwitz, Über der Komposition der quadratischer Formen, Math. Ann. 88 (1923), 1-25. 
5. I. JAMES AND E. THOMAS, An approach to the enumeration problem for non-stable vector bundles, J. Math. Mech. 14 (1965), 485-506.

6. K. Y. LAM AND P. Y. H. YIU, Sums of squares formulae near the Hurwitz-Radon range, Contemp. Math. 58 (1987), 51-56.

7. E. Looljenga, A note on polynomial isolated singularities, Nederl. Akad. Wetensch. Proc. A 74, Indag. Math. 33 (1971), 418-421.

8. J. W. MILnOR, Singular points of complex hypersurfaces (Princeton University Press, 1968).

9. B. Perron, Le noeud 'huit' est algebrique reel, Invent. Math. 65 (1981/82), 441-451.

10. J. RADON, Lineare scharen orthogonalen Matrizen, Abh. Math. Sem. Univ. Hamburg 1 (1922), 1-14.

11. E. G. ReEs, Linear spaces of real matrices of given rank, Contemp. Math. 188 (1995), 219-229.

12. E. G. Rees, Linear spaces of real matrices, in Proc. A. C. Aitken Centenary Conf., Dunedin, 1995; Otago Conf. Series 5 (ed. L. Kavalieris, F. C. Lam, L. A. Roberts and J. A. Shanks), pp. 311-320 (University of Otago Press, 1996).

13. J. A. SeAde, Fibred links and a construction of real singularities via complex geometry, Bol. Soc. Brasil. Mat. (NS) 27 (1996), 199-215.

14. T. YoshIDA, On the vector bundles $m \xi_{n}$ over real projective spaces, J. Sci. Hiroshima Univ. Ser. A-I Math. 32 (1968), 5-16. 